\title{
Virtual modelling of novel applicator prototypes for cervical cancer brachytherapy
}

\author{
Primoz Petric ${ }^{1,2}$, Robert Hudej², Noora Al-Hammadi ${ }^{1}$, Barbara Segedin² \\ ${ }^{1}$ National Center for Cancer Care and Research, Hamad Medical Corporation, Doha, Qatar \\ 2 Institute of Oncology Ljubljana, Ljubljana, Slovenia
}

Radiol Oncol 2016; 50(4): 433-441

Received 1 July 2016

Accepted 29 August 2016

Correspondence to: Primož Petrič, M.D., M.Sc., National Center for Cancer Care and Research, Hamad Medical Corporation, P.O. Box 3050, Doha, Qatar; Phone:+ 974 44397720; E-mail: pp.visoko@gmail.com

Disclosure: The presented work is a part of the GEC ESTRO Gyn Applicator Development Project and was partially funded by a grant from Varian Medical Systems Inc.

Background. Standard applicators for cervical cancer Brachytherapy (BT) do not always achieve acceptable balance between target volume and normal tissue irradiation. We aimed to develop an innovative method of Targetvolume Density Mapping (TDM) for modelling of novel applicator prototypes with optimal coverage characteristics. Patients and methods. Development of Contour-Analysis Tool 2 (CAT-2) software for TDM generation was the core priority of our task group. Main requests regarding software functionalities were formulated and guided the coding process. Software validation and accuracy check was performed using phantom objects. Concepts and terms for standardized workflow of TDM post-processing and applicator development were introduced.

Results. CAT-2 enables applicator-based co-registration of Digital Imaging and Communications in Medicine (DICOM) structures from a sample of cases, generating a TDM with pooled contours in applicator-eye-view. Each TDM voxel is assigned a value, corresponding to the number of target contours encompassing that voxel. Values are converted to grey levels and transformed to DICOM image, which is transported to the treatment planning system. Iso-density contours (IDC) are generated as lines, connecting voxels with same grey levels. Residual Volume at Risk $(R V R)$ is created for each IDC as potential volume that could contain organs at risk. Finally, standard and prototype applicators are applied on the TDM and virtual dose planning is performed. Dose volume histogram (DVH) parameters are recorded for individual IDC and RVR delineations and characteristic curves generated. Optimal applicator configuration is determined in an iterative manner based on comparison of characteristic curves, virtual implant complexities and isodose distributions.

Conclusions. Using the TDM approach, virtual applicator prototypes capable of conformal coverage of any target volume, can be modelled. Further systematic assessment, including studies on clinical feasibility, safety and effectiveness are needed before routine use of novel prototypes can be considered.

Key words: cervical cancer; brachytherapy; applicators

\section{Introduction}

Brachytherapy (BT) is an essential component of treatment for locally advanced cervical cancer. Historically, X ray-based BT has been used, resulting in suboptimal disease control and toxicity rates, especially in advanced tumours. ${ }^{1-5}$ Currently, image guided adaptive brachytherapy (IGABT) is being increasingly implemented worldwide. This modern technique is based on geometric individualization of the BT implant, sectional imaging, computerized treatment planning and remote afterloading technology. ${ }^{6}$ The concept of IGABT enables tight control over dose distribution in the tissues and personalized adaptation of treatment according to individual tumour size, topography and relation to the organs at risk (OAR). Consequently, dose escalation to the target volume is achieved, 
while respecting OAR dose constraints. Dosimetric advantages of IGABT over conventional 2D BT are well documented and are reflected in superior clinical outcomes. ${ }^{7-16}$

Even with the most sophisticated treatment planning technology, optimal placement of source channels in the target volume remains a precondition for the success of BT. The ability to compensate for inadequacies of suboptimal insertion by optimization of source dwell-times and dwellpositions is limited. Standard intracavitary (IC) applicators for BT of cervical cancer do not always enable an acceptable balance between the coverage of the target volume and dose to the OAR. ${ }^{17}$ In patients with unfavourable tumour topography and/or gross residual disease extending beyond the reach of standard dose distribution, parts of the tumour cannot be covered with the prescribed dose without exceeding OAR tolerance. This leads to dosimetric cold spots in the tumour that predispose to treatment failure. ${ }^{18,19}$ Recently developed intracavitary and interstitial (IC/IS) applicators for transvaginal placement of parallel parametrial needles allow for moderate lateral displacement of prescribed isodose. ${ }^{20-23}$ While this leads to favourable therapeutic ratio in majority of cases, there is substantial proportion of challenging tumours which require alternative techniques for optimal outcome. Systematic assessment of dosimetric characteristics of currently available IC and IC/IS applicators or novel prototypes has not been performed so far. Theoretically, an ideal BT applicator would enable standardized and reproducible insertion of the IC and IS channels in optimal geometries, allowing for complete coverage of any tumour encountered in clinical practice. In this manuscript we present an innovative approach to evidence-based applicator development, using the method of Target-volume Density Mapping (TDM). TDM methodology was consequently applied in multi-institutional setting and promising preliminary outcome was presented. ${ }^{24}$ Publication of the final results of this analysis is pending.

We conducted this study as a part of a wider applicator development project, performed by the international Gyn GEC ESTRO network. The work presented here is entirely technical, focusing merely on the software development and specification of related methodology. No patient information was used during this work. The developed methodology was consequently used in substudies on patients, included in the multi-institutional EMBRACE and retroEMBRACE protocols. These protocols were approved by the institutional re- view boards / ethical committees of participating centres. Promising preliminary results of this analysis were presented, while the publication of the final results is pending. ${ }^{24}$ In the present manuscript, only the theoretical concept of software-based methodology for TDM generation is detailed.

\section{Methods}

Multidisciplinary task group from the Institute of Oncology Ljubljana and the University of Primorska, Faculty of Mathematics, was formed in 2009 to propose methodological framework for evidence based applicator modelling. The group consisted of an experienced radiation oncologist, a medical physicist and a computer scientist. The activities of the group were arbitrarily divided into (1) TDM software development and (2) Applicator modelling.

\section{Development of software for target density mapping}

Development of TDM software was identified as core priority of the task group. The coding process reflected three main domains of requests regarding application functionalities:

Individual input data should consist of singlecase DICOM(Digital Imaging and Communications in Medicine) files containing target and applicator structures.

Sequential import of DICOM data-sets from multiple cases should be enabled, followed by rigid co-registration of structures, using the IC applicator as the reference.

Software output should be an object in DICOM format, containing pooled information from individual cases.

Validation and geometric accuracy check of the software application was based on the import and processing of a series of phantom DICOM datasets. The phantom sets consisted of virtual tandem \& ring applicators and structures of known shapes, sizes and topographic relations with the applicator.

\section{Modelling of virtual applicator prototypes}

Standardized model for quantitative analysis of dosimetric effects of existent and prototype applicators on the TDM was developed. This task included the definition of concepts, terms and workflow for post-processing of the TDM. Applying 
these definitions, the workflow for computer based modelling of applicator prototypes was developed.

\section{Results}

\section{Software for target density mapping}

Dedicated software (Contour Analysis Tool 2 - CAT 2) was developed by the task group. The principles of CAT 2 application are outlined in Figure 1. The method is based on sequential import of DICOM structure files from a representative sample of individual cases, containing the target volume and the tandem \& ring applicator. CAT 2 performs rigid co-registration of the imported structures, based on the applicator as the frame of reference. This implies rotational and translational shifts of the target volume and the applicator, resulting in alignment of the (1) centre of the ring, (2) tandem and ring axes and (3) axis connecting the centre of the ring with the first ring-source position. Consequently, TDM is created as a hybrid data set, containing pooled information from a sample of cases. Individual target volumes in the TDM are aligned to the single reference applicator, maintaining unchanged relative topography to the applicator axes as in the original data set. The differences in tandem length and ring diameter are not taken into account during co-registration process. Each voxel of the TDM is assigned a Target Density Value (TDV), corresponding to the number of target volume contours that encompass that voxel. Finally, TDVs are converted to grey levels and the data set is transformed to DICOM image, containing the reference applicator and the TDM in an "applicator-eye-view".

\section{Modelling of virtual applicator prototypes}

This workflow starts with the import of the TDM DICOM object to the treatment planning system. Post-processing steps are summarized below:

\section{Generation of Iso-Density Contours and Residual Volumes at Risk}

Iso-density contours (IDC) are generated using the auto-segmentation functionality of the treatment planning system. IDCs are defined as delineations, connecting voxels with the same TDV and are labelled as percentage of encompassed TDVs. Labels of individual IDCs are calculated by using the following formula:

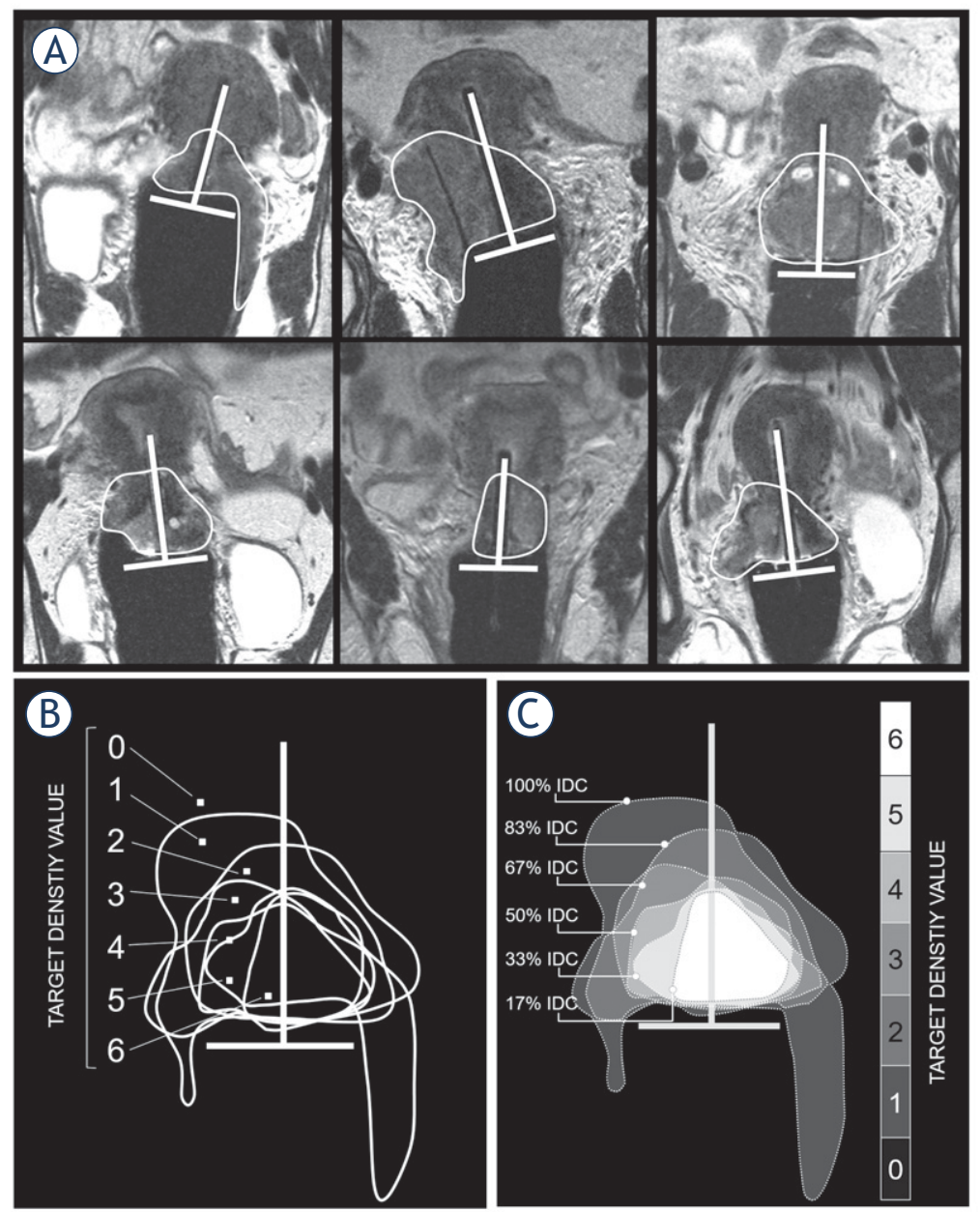

FIGURE 1. Principles of Target-volume Density Map (TDM) generation and postprocessing on an example of 6 cervical cancer cases. (A) Contours of high risk clinical target volumes $\left(\mathrm{CTV}_{\mathrm{HR}}-\right.$ thin white lines) are shown on mid-coronal T2 weighted MRI with the applicator in place. Source channels are depicted as thick white lines. (B) CAT 2 generates the TDM by rigid co-registration of individual $C_{T V} V_{H R} s$ on a reference applicator. TDM voxels are assigned target density values, corresponding to the number of encompassing $\mathrm{CTV}_{\mathrm{HR}}$ s. These values are transformed to grey levels. (C) Resulting TDM DICOM image is exported to treatment planning system where isodensity contours (IDC) are auto-segmented (white dotted lines).

$$
\mathrm{IDC}_{\mathrm{TDV}}=\frac{\mathrm{TDV}_{\max }-\mathrm{TDV}+1}{\mathrm{TDV}_{\max }} \times 100[\%]
$$

For example, the label of outermost IDC in Figure $1 \mathrm{C}\left(\mathrm{TDV}=1\right.$ and $\left.\mathrm{TDV}_{\max }=6\right)$ is:

$$
\mathrm{IDC}_{1}=\frac{6-1+1}{6} \times 100=100 \%
$$

And the label of innermost IDC in Figure 1C $\left(\mathrm{TDV}=6\right.$ and $\left.\mathrm{TDV}_{\max }=6\right)$ is:

$$
\mathrm{IDC}_{1}=\frac{6-6+1}{6} \times 100=17 \%
$$

Therefore, outermost IDC encompasses voxels with TDVs of 1 to 6 (100\% of TDVs) while the in- 
nermost IDC encompasses only the voxels with TDV of 6 (17\% of TDVs) (Figure 1C). Following auto-segmentation of IDCs, residual volume at risk $\left(\mathrm{RVR}_{\mathrm{TDV}}\right)$ is generated for each $\mathrm{IDC}_{\mathrm{TDV}}$, using the Boolean operators of the treatment planning system. $\mathrm{RVR}_{\mathrm{TDV}}$ is defined as volume of virtual patient minus the volume of the $\mathrm{IDC}_{\mathrm{TDV}}$. It corresponds to the potential volume in which the tissues and OAR could be located in a hypothetical patient with the target volume of IDC $_{\mathrm{TDV}}$.

\section{Application of the standard treatment plan on TDM}

Conventional treatment plan, based on the IC applicator with standard loading and dose specification at Manchester point A is applied to the TDM (Figure 2A). Dose volume histogram (DVH) pa-
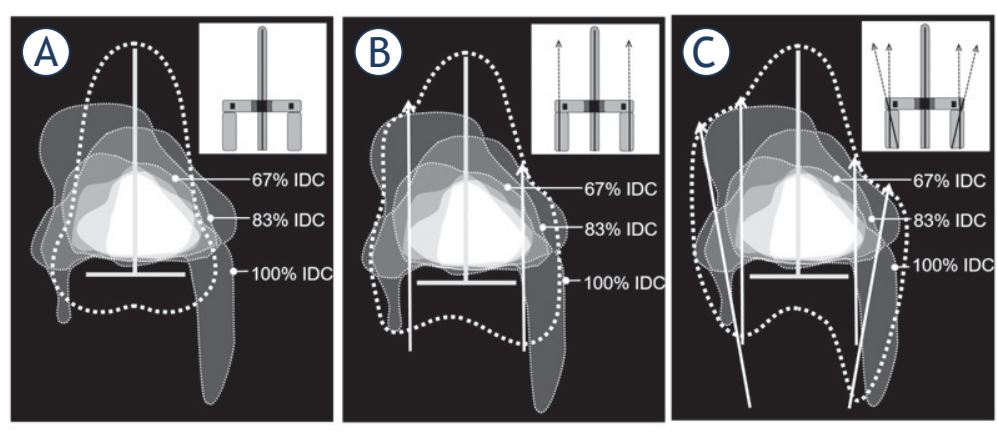

(D)

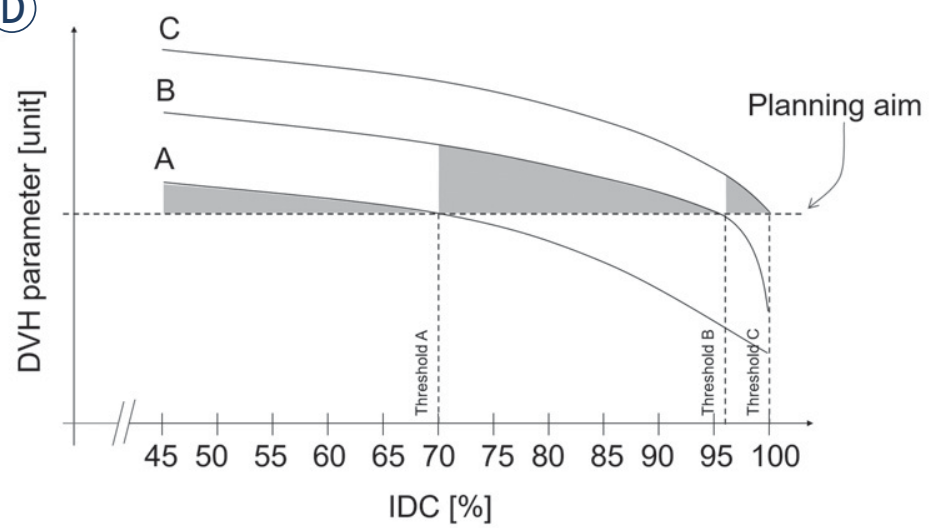

FIGURE 2. Schematic representation of applicator modelling based on our theoretical example of Target-volume Density Map (TDM). Above: Three virtual applicators are reconstructed on the TDM. Dose distribution is optimized based on a set of specific planning aims for the isodensity contours (IDC) and for residual volumes at risk (RVR). The optimized prescription isodoses for individual applicators are shown as thick dotted lines. (A) Standard intracavitary applicator: limited possibility for optimization. The planning aim is achieved for $\approx 70 \%$ IDC. (B) Combined intracavitary and interstitial applicator with parallel parametrial needles (Vienna-type): planning aim is achieved for $\approx 95 \%$ IDC. (C) Combined intracavitary and interstitial applicator with parallel and oblique parametrial needles: planning aim as achieved for $\approx 100 \%$ IDC. (D) Characteristic curves for the IDC of the three applicator types. RVR curves are not shown. Shaded areas under characteristic curves represents IDC ranges in which certain applicator is able to achieve the planning aim. Arbitrary planning aim and applicator thresholds are marked by dotted lines. rameters are recorded for individual $\mathrm{IDC}_{\mathrm{TDV}} \mathrm{S}$ and $\mathrm{RVR}_{\mathrm{TDV}} \mathrm{s}$. Recommended minimal set of DVH parameters for IDC $_{\mathrm{TDV}}$ includes volume receiving the prescribed dose (V100) and dose received by the $90 \%$ and $98 \%$ (D90 and D98) of the IDC $\mathrm{TDV}_{\text {volume. }}$ For $\mathrm{RVR}_{\mathrm{TDV}}$, minimum doses received by $2 \mathrm{~cm}^{3}$ of the most irradiated $\mathrm{RVR}_{\mathrm{TDV}}(\mathrm{D} 2 \mathrm{Cc})$ are recorded. Characteristic curves for the IC applicator with standard loading are generated by plotting the DVH parameters against IDC $_{\mathrm{TDV}}$ and $\mathrm{RVR}_{\mathrm{TDV}}$ levels (Figure 2D).

\section{Modelling of class solution IC+IS applicators}

During this step of the process, characteristic curves are used to assess the limitations of standard IC plans. TDV threshold at which the standard plan fails to meet the planning aims for IDC and dose constraints for RVR are identified (Figure 2). Consequently, qualitative assessment of dose distributions is performed for treatment plans above the threshold. This includes topographical analysis of under-dosed and over-dosed regions of IDC and RVR, respectively. Based on the topographic analysis, virtual IS channels are added to the standard IC applicator to achieve optimal implant geometry for the corresponding IDC and RVR (Figure 2B and $2 \mathrm{C}$ ). Next, source dwell-positions and dwelltimes inside the IC and IS channels are adjusted to optimize the dose distribution. These steps are repeated in an iterative manner until class-solution applicators, capable of covering complete TDM are modelled. Determination of optimal implant geometry and loading and specification of planning aims (IDC) and dose constraints (RVR) may vary between or within planners, leading to various applicator solutions. For the purpose of this project, we applied our institutional standard pre-planning and optimization procedure which was described recently. ${ }^{25}$ Finally, characteristic curves of the modelled prototypes are generated and compared (Figure 2D).

\section{Discussion}

In our present project, we developed CAT 2 software for TDM generation and related post-processing methodology. Our method enables evidencebased modelling of novel IC/IS applicator prototypes and in-silico testing of existent devices. The proposed approach is based on analysis of pooled spatial information of target volumes from a representative sample of patients in the "applicator-eye view". 
Lessons learned from historical evolution of applicators are essential for optimal utilization of modern development strategies, including our TDM method. Historical Paris and Stockholm techniques date back approximately 100 years. ${ }^{26-28}$ The applicators consisted of a rubber tandem and a pair of colpostats (Paris technique) or flat vaginal box (Stockholm method), pre-loaded with ${ }^{226} \mathrm{Ra}$. In the 1930's, the Manchester system was developed, introducing the point $\mathrm{A}$ and the applicator which was geometrically similar to its predecessors. ${ }^{29}$ Nowadays, fixed geometry of uterine tandem and vaginal components (ovoids, cylinders or ring) enables reproducible insertion and reliable treatment using small sealed sources and afterloading technology. Standard loading patterns of modern applicators mimic the historical patterns based on milligram-hours of ${ }^{226} \mathrm{Ra}$ tradition. ${ }^{30}$ In topographically unfavourable tumours, optimization of loading can expand the prescribed dose for up to approximately $4 \mathrm{~mm}$ at the point A level. ${ }^{17}$ Target volumes that extend beyond this region and cases with unfavourable OAR topography pose a challenge to standard IC techniques. Applicators with static shielding in the ovoids were developed to improve the therapeutic ratio by reducing the irradiation of the OAR and their dosimetric impact was studied extensively. ${ }^{31-34}$ Use of shielded devices outside the context of IGABT and model-based dose calculation algorithms may result in unintentional shielding of the target volume and uncertainties regarding the OAR doses. ${ }^{32}$ Emerging technologies, including static adjustable shielding and dynamic modulated BT with rotating paddle, can achieve highly conformal dose distributions in large targets of complex shapes. ${ }^{35-37}$ However, when used in pure IC BT, directional modulation of intensity may lead to excessively high doses near the applicator along the emission window axis. ${ }^{36}$ These techniques need to stand the test of further studies before their routine implementation. Dynamic shielding may play an important role in the future, especially if applied in combination with IC/IS insertion technique. ${ }^{37}$ In summary, the exciting technological developments which we have witnessed over the past century failed to overcome the inherent physical limitations of IC BT.

Manufacturing of non-ferromagnetic applicators allowed for implementation of MRI based IGABT. ${ }^{38-41}$ This can be regarded as the single most important advancement in the field of IC applicator development over last decades. IGABT offered an unprecedented opportunity to assess limitations of standard IC applicators and design im- proved IC/IS prototypes. TDM methodology described in this manuscript is based on estimation of the impact of individual applicator adjustments on a single "virtual patient", containing pooled information from a sample of patients. In contrast, the traditional paradigm of empirical development is based on analysis of applicator limitations in individual cases, followed by iterative process of technical improvements. Using the traditional paradigm, novel IC/IS applicators were developed recently. Dimopoulos et al. introduced the Vienna tandem $\mathcal{E}$ ring applicator in which the ring serves as template for insertion of parallel needles into the parametria. ${ }^{20}$ In their first report, 170 needles were inserted in 22 patients. The mean depth of insertion was only $37+/-7 \mathrm{~mm}$ and the mean difference between planned and actual needle tip positions was sub-millimetric. Due to the use of blunt needles and short needle tracks, no organ perforations were identified and only $8.8 \%$ of the needles were outside the $\mathrm{CTV}_{\mathrm{HR} .}{ }^{20}$ Tandem \& ovoids applicator underwent similar evolution, resulting in the Utrecht applicator with comparable geometric, clinical and dosimetric characteristics. ${ }^{22,23}$ IGABT with these application techniques enables moderate expansion and individual shaping of the isodose distribution, leading to improved dosimetric and clinical outcome. ${ }^{13,14,17,22,23}$ However, tumours with major extra-cervical infiltration at time of BT that extends beyond dosimetric reach of these applicators remain to pose a special challenge (i.e. infiltration of sacrouterine ligaments and distal parametria).

TDM-based methodology allows for objective quantification of IC and IC/IS applicator characteristics. By studying the impact of implant-geometry adjustments, prototypes of novel applicators that are theoretically capable of covering any target volume can be generated. Our method has been endorsed by the Gynaecological GEC ESTRO Network and preliminary results of its implementation in a multi-centre setting have been published in abstract form. ${ }^{24}$ In this study, $\mathrm{CTV}_{\mathrm{HR}}$ delineations from a representative sample of 264 patients were used to generate a TDM. Standard IC and Vienna IC/IS applicators achieved coverage of around 60\% and 95\% IDC, respectively. Prototypes, able of covering the remaining $5 \%$ of most challenging IDCs were proposed by adding virtual oblique needles to the Vienna applicator. ${ }^{24}$ The impact of applicator geometries and dose optimization on RVR envelopes was not assessed in this preliminary report.

In the final analysis of this study, which is currently underway, two dose optimization models 
will be applied: standard and optimized. (1) In the standard model of dose planning, no RVRs will be assumed around the IDCs and no RVR-based optimization / reporting will be performed. This model therefore represents the most favourable theoretical scenario in terms of IDC dose-coverage. (2) In the optimized model, each IDC will be entirely surrounded by an RVR envelope and achieving the dose constraints for the RVR will be the absolute priority of dose optimization process. Optimized model is therefore the theoretical worst case scenario in terms of IDC coverage and it is practically never encountered in real clinical practice. In optimized model, the reported D2cc parameters for all the RVR envelopes will be the same, reflecting the RVR dose constraints. The utility of the proposed two-model approach is as follows: the two extreme planning approaches are the limiting scenarios, between which the actual clinical situations are expected. Consequently, the area between the two limiting IDC characteristic curves can be regarded as the "useful area" of a specific applicator. We believe that the development of new applicator prototypes, based on the TDM method will lead to further testing in clinical studies and finally commercial availability of safe, feasible and dosimetrically optimal devices, capable of covering the BT target volumes in any clinical situation. It can be expected that this will translate into further improved clinical outcomes of locally advanced cervical cancer management.

The emerging technology of personalized 3D printing of single-use IC/IS BT applicators is an exciting alternative to commercially available devices. ${ }^{42}$ Individual tailoring of the applicator size, shape and source channel geometry according to patient's anatomy and tumour topography can be considered as the ultimate method to adaptive BT insertion. Advanced level of expertise, routine availability of pre-BT MRI, access to 3D printer and dedicated clinical time are required for implementation of 3D printing approach. Thus, standard IC/ IS applicators remain to play an important role, especially in clinical settings with high incidence of cervical cancer and limited resources, making the TDM method presented here highly relevant.

In our method, the differences in tandem length and ring diameter are not taken into account during co-registration process, leading to a minimal overlap between the central parts of some target volumes with the reference applicator. Since central voxels are located in vicinity of the sources and high dose regions, this inconsistency bears minimal significance and is of limited interest for ap- plicator development. The relevance of applicator development in the context of IGABT depends on accuracy of contouring of the target volume and organs at risk. There is a growing body of evidence that contouring uncertainties may be the weakest link in the process of IGABT. ${ }^{43}$ Since our TDM methodology relies on import of individual target volume delineations, contouring uncertainties can undermine the validity of TDM-based applicator prototyping. Therefore, quality assurance of imported contours is essential for clinically relevant utilization of our method. There are three major aspects that need to be considered in this regard: (1) selection of target volume for TDM generation, (2) experience of radiation oncologists and (3) imaging modality for target volume delineation.

\section{Selection of target volume for target density mapping}

$\mathrm{CTV}_{\mathrm{HR}}$ was recently identified as the most robust target volume for dose prescription and optimization in cervical cancer IGABT. ${ }^{44}$ When comparing delineations of ten observers with the expert consensus contours, the mean conformity index for the GTV, CTV ${ }_{\text {IR }}$ and $\mathrm{CTV}_{\mathrm{HR}}$ was $0.58,0.68$ and 0.72 , respectively. Corresponding mean inter-delineation distances were $4.2+/-3.5 \mathrm{~mm}, 5.2+/-5.6 \mathrm{~mm}$ and 3.8 +/- $3.4 \mathrm{~mm}$, respectively. ${ }^{44}$ Other published studies revealed similar degree of inter-observer variation for the $\mathrm{CTV}_{\mathrm{HR} \text {. }}{ }^{45-47}$ and confirmed more favourable results for $\mathrm{CTV}_{\mathrm{HR}}$ when compared with GTV and $\mathrm{CTV}_{\mathrm{IR}}{ }^{45}$ In conclusion, we recommend using $\mathrm{CTV}_{\mathrm{HR}}$ as the target structure for TDM generation.

\section{Experience of radiation oncologists}

$\mathrm{CTV}_{\mathrm{HR}}$ contouring is a challenging procedure with a long learning curve. ${ }^{43}$ The observers in published inter-observer studies on cervical cancer IGABT were experts, participating in the EMBRACE protocol (IntErnational study on MRI-guided BRAchytherapy in locally advanced CErvical cancer) or contouring instructors. ${ }^{44-48}$ GEC ESTRO recommendations were strictly applied in these studies. ${ }^{38,50}$ In spite of the favourable circumstances, the magnitude of "residual" uncertainties at expert level was substantial and was reflected in clinically relevant uncertainties of the reported DVH parameters. ${ }^{44,48}$ Therefore, complete elimination of contouring uncertainties from clinical practice is not-realistic. This needs to be taken into account when interpreting the results of studies and is one of the major inherent limitations of the TDM 
method presented here. To ensure optimal validity of the TDM method, the imported contours should ideally originate from clinical studies with expert-based peer review or plausibility checking for quality control.

\section{Imaging modality}

MRI is gold standard for cervical cancer IGABT and GEC ESTRO recommendations on various aspects of its use in IGABT were published. ${ }^{38-41}$ Excellent clinical results of mono- and multi-institutional studies can be regarded as indirect confirmation of validity of the concepts and terms, defined in these recommendations. ${ }^{9-16}$ While guidelines for CT-based contouring recently became available, physical limitations of CT for soft tissue depiction remain a major impediment. ${ }^{50}$ Not surprisingly, inter-observer studies on contouring variation demonstrate superior results for MRI-based delineation when compared with CT-based approach. ${ }^{44,51-54}$ Ultrasound and PET CT may add complementary information during delineation, but their role as eventual independent modality for contouring remains to be defined. In summary, TDM-guided applicator prototyping should be based on target volumes, delineated on MRI.

\section{Conclusions}

We developed methodology for applicator modelling based on pooled analysis of target volumes in reference applicator-eye-view. The system was validated using the phantom geometries. Recommendations to minimize uncertainties resulting from contouring variation were proposed. Using our method, virtual applicator prototypes capable of covering any target volume can be developed. Computer-based modelling represents the basic step in development of applicator prototypes which need to undergo further systematic assessment, including studies on clinical feasibility and safety. Our methodology was applied in multi-institutional setting and promising preliminary outcome published. ${ }^{24}$ Final analysis of the data is currently underway.

\section{Acknowledgements}

The authors are grateful to Mr. Omar Hanuna, Institute of Oncology of Ljubljana for his valuable contribution to data collection, analysis and support during manuscript preparation. We are grateful to Prof. Peter Rogelj, PhD (University of Primorska, Faculty of Mathematics, Natural Sciences and Information Technologies, Koper, Slovenia) for the time and effort, invested in software development. We highly appreciate contributions of the Gyn GEC ESTRO Network, in particular the teams of Medical University of Vienna and Aarhus University Hospital, for their contribution to the presented work.

\section{References}

1. Gerbaulet A, Pötter R, Haie-Meder C. Cervix cancer. In: Gerbaulet A, Pötter $\mathrm{R}$, Mazeron JJ, Meertens H, Van Limbergen E, editors. The GEC ESTRO handbook of brachytherapy. Brussels: European Society of Therapeutic Radiology and Oncology; 2002. p 300-63.

2. Horiot JC, Pigneux J, Pourquier H. Radiotherapy alone in carcinoma of intact uterine cervix according to Fletcher guidelines: a French cooperative study of 1383 cases. Int J Radiat Oncol Biol Phys 1988; 14: 605-11.

3. Perez CA, Breaux S, Bedwinek JM, Madoc-Jones H, Camel HM, Purdy JA, et al. Radiation therapy alone in the treatment of carcinoma of the uterine cervix II. Analysis of complications. Cancer 1984; 54: 35-46.

4. Pernot M, Hoffstetter S, Peiffert D, Carolus JM, Guillemin F, Verhaeghe JL, et al. Statistical study of a series of 672 cases of carcinoma of the uterine cervix. Results and complications according to age and modalities of treatment. Bull Cancer 1995; 82: 568-81.

5. Pötter R, Knocke TH, Fellner C, Baldass M, Reinthaller A, Kucera A. Definitive radiotherapy based on HDR brachytherapy with Iridium-192 in cervix cancer-report on the recent Vienna university hospital experience (1993-1997) compared to the preceding period, referring to ICRU 38 recommendations. Cancer Radiother 2000; 4: 159-172.

6. Pötter R, Kirisits C, Fidarova EF, Dimopoulos JC, Berger D, Tanderup K Lindegaard JC. Present status and future of high-precision image guided adaptive brachytherapy for cervix carcinoma. Acta Oncol 2008; 47: 1325-36.

7. Lindegaard JC, Tanderup K, Nielsen SK, Haack S, Gelineck J. MRI-Guided 3D Optimization Significantly Improves DVH Parameters of Pulsed-Dose-Rate Brachytherapy in Locally Advanced Cervical Cancer. Int J Radiat Oncol Biol Phys 2008; 71: 756-64.

8. De Brabandere M, Mousa AG, Nulens A, Swinnen A, Van Limbergen E. Potential of dose optimisation in MRI-based PDR brachytherapy of cervix carcinoma. Radiother Oncol 2008; 88: 217-26.

9. Pötter R, Dimopoulos J, Georg P, Lang S, Waldhäusl C, Wachter-Gerstner $\mathrm{N}$, et al. Clinical impact of MRI assisted dose volume adaptation and dose escalation in brachytherapy of locally advanced cervix cancer. Radiother Oncol 2007; 83: 148-55.

10. Pötter R, Georg P, Dimopoulos JC, Grim MM, Berger D, Nesvacil N, et al. Clinical outcome of protocol based image (MRI) guided adaptive brachytherapy combined with 3D conformal radiotherapy with or without chemotherapy in patients with locally advanced cervical cancer. Radiother Oncol 2011; 100: 116-23.

11. Haie-Meder C, Chargari C, Rey A, Dumas I, Morice P, Magné N. MRI-based low dose-rate brachytherapy experience in locally advanced cervical cancer patients initially treated by concomitant chemoradiotherapy. Radiother Oncol 2010; 96: 161-5.

12. Ribeiro I, Janssen H, De Brabandere M, Nulens A, De Bal D, Vergote I, Van Limbergen E. Long term experience with 3D image guided brachytherapy and clinical outcome in cervical cancer patients. Radiother Oncol 2016; [Epub ahead of print] PMID: 27157510

13. Fokdal L, Sturdza A, Mazeron R, Haie-Meder C, Tan LT, Gillham C, et al. Image guided adaptive brachytherapy with combined intracavitary and interstitial technique improves the therapeutic ratio in locally advanced cervical cancer: Analysis from the retroEMBRACE study. Radiother Oncol 2016; doi: 10.1016/j.radonc.2016.04.016. [Epub ahead of print] PMID: 27113795 
14. Sturdza A, Pötter R, Fokdal LU, Haie-Meder C, Tan LT, Mazeron R, Petric $P$, et al. Image guided brachytherapy in locally advanced cervical cancer: Improved pelvic control and survival in RetroEMBRACE, a multicenter cohort study. Radiother Oncol 2016; [Epub ahead of print] PMID: 27134181

15. Kirchheiner K, Pötter R, Tanderup K, Lindegaard JC, Haie-Meder C, Petric $P$ et al. EMBRACE Collaborative Group. Health-related quality of life in locally advanced cervical cancer patients after definitive chemoradiation therapy including image guided adaptive brachytherapy: An analysis from the EMBRACE study. Int J Radiat Oncol Biol Phys 2016; 94: 1088-98.

16. Castelnau-Marchand P, Chargari C, Maroun P, Dumas I, Del Campo ER, Cao $\mathrm{K}$, et al. Clinical outcomes of definitive chemoradiation followed by intracavitary pulsed-dose rate image-guided adaptive brachytherapy in locally advanced cervical cancer. Gynecol Oncol 2015; 139: 288-94.

17. Kirisits C, Pötter R, Lang S, Dimopoulos J, Wachter-Gerstner N, Georg D. Dose and volume parameters for MRI based treatment planning in intracavitary brachytherapy of cervix cancer. Int J Raddiat Oncol Biol Phys 2005 62: 901-11.

18. Dimopoulos JC, Lang S, Kirisits C, Fidarova EF, Berger D, Georg P, et al Dose-volume histogram parameters and local tumor control in magnetic resonance image-guided cervical cancer brachytherapy. Int J Radiat Oncol Biol Phys 2009; 75: 56-63.

19. Schmid MP, Kirisits C, Nesvacil N, Dimopoulos JC, Berger D, Pötter R. Local recurrences in cervical cancer patients in the setting of image-guided brachytherapy: a comparison of spatial dose distribution within a matchedpair analysis. Radiother Oncol 2011; 100: 468-72.

20. Dimopoulos JCA, Kirisits C, Petric P, Georg P, Lang S, Berger D, Pötter R. The Vienna applicator for combined intracavitary and interstitial brachytherapy of cervical cancer: clinical feasibility and preliminary results. Int J Radiat Oncol Biol Phys 2006; 66: 83-90.

21. Kirisits C, Lang S, Dimopoulos J, Berger D, Georg D, Pötter R. The Vienna applicator for combined intracavitary and interstitial brachytherapy of cervical cancer: design, application, treatment planning, and dosimetric results. Int Radiat Oncol Biol Phys 2006; 65: 624-30.

22. Nomden CN, De Leeuw AAC, Moerland MA, Roesink JM, Tersteeg RJ, Jürgenliemk-Schulz I. Clinical use of the Utrecht applicator for combined intracavitary/interstitial brachytherapy treatment in locally advanced cervical cancer. Int J Radiat Oncol Biol Phys 2012; 82: 1424-30.

23. Jürgenliemk-Schulz IM, Tersteeg RJHA, Roesink JM, Bijmolt S, Nomden Cn, Moerland MA, de Leeuw AA. MRI-guided treatment planning optimisation in intracavitary or combined intracavitary/interstitial PDR brachytherapy using tandem ovoid applicators in locally advanced cervical cancer. Radiother Oncol 2009; 93: 322-30.

24. Petric P, Hudej R, Rogelj P, Lindegaard J, Tanderup K, Kirisits C, et al. Frequency-distribution mapping of HR CTV in cervix cancer: possibilities and limitations of existent and prototype applicators. Radiother Oncol 2010 96(Suppl 1): S70

25. Petric P, Hudej R, Hanuna O, Marolt P, Al-Hammadi N, Riyas MP, Segedin B. MRI-assisted cervix cancer brachytherapy pre-planning, based on application in paracervical anaesthesia: final report. Radiol Oncol 2014; 48: 293-300.

26. Pötter R, Van Limbergen E, Wambersie A. Reporting in Brachytherapy: Dose and Volume Specification. In: Gerbaulet A, Pötter R, Mazeron JJ, Meerten $\mathrm{H}$, Van Limbergen E, editors. The GEC ESTRO Handbook of Brachytherapy. Brussels: European Society of Therapeutic Radiology and Oncology; 2002. p. $153-215$.

27. Heymann J. The so-called Stockholm method and the results of treatment of uterine cancer at Radiumhemmet. Acta Radiol 1935; 16: 129-48.

28. Kottmeier HL. Surgical and radiation treatment of carcinoma of the uterine cervix. Experience by the current individualized Stockholm technique. Acto Obstet Gynecol Scand 1964; 43: 1-48.

29. Tod M, Meredith WJ. Treatment of cancer of the cervix uteri: A revised Manchester method. Br J Radiol 1953; 26: 252-7.

30. Jamema SV, Kirisits C, Mahantshetty U, Trnkova P, Deshpande DD, Shrivastava SK, Pötter R. Comparison of DVH parameters and loading patterns of standard loading, manual and inverse optimization for intracavitary brachytherapy on a subset of tandem/ovoid cases. Radiother Oncol 2010; 97: 501-6.
31. Hira M, Podgorsak MB, Jaggernauth W, Malhotra HK. Measurement of dose perturbation around shielded ovoids in high-dose-rate brachytherapy. Brachytherapy 2011; 10: 232-41.

32. Gifford KA, Horton JL, Pelloski CE, Jhingran A, Court LE, Mourtada F, Eifel P. A three-dimensional computed tomography-assisted Monte Carlo evaluation of ovoid shielding on the dose to the bladder and rectum in intracavitary radiotherapy for cervical cancer. Int J Radiat Oncol Biol Phys 2005; 63: 615-21.

33. Price MJ, Gifford KA, Horton JL, Eifel PJ, Gillin T, Lawyer AA, Mourtada F. Monte Carlo model for a prototype CT-compatible, anatomically adaptive, shielded intracavitary brachytherapy applicator for the treatment of cervical cancer. Med Phys 2009; 36: 4147-55.

34. Steggarda MJ, Moonen LM, Damen EM, Lebesque JV. An analysis of the effect of ovoid shields in a selectron-LDR cervical applicator on dose distribution in rectum and bladder. Int J Radiat Oncol Biol Phys 1997; 39: 237-45.

35. Han DY, Webster MJ, Scanderberg DJ, Yashar C, Choi D, Song B, et al Direction-modulated brachytherapy for high-dose-rate treatment of cervical cancer. I: theoretical design. Int J Radiat Oncol Biol Phys 2014; 89: 666-73.

36. Liu Y, Flynn RT, Kim Y, Yang W, Wu X. Dynamic rotating-shield brachytherapy. Med Phys 2013; 40: 12703

37. Adams QE, Xu J, Breitbach EK, Li X, Enger SA, Rockey WR, et al. Interstitial rotating shield brachytherapy for prostate cancer. Med Phys 2014; 41: 051703.

38. Haie-Meder C, Pötter R, Van Limbergen E, Briot E, De Brabandere M, Dimopoulos J, et al. Recommendations from Gynaecological (GYN) GECESTRO Working Group (I): concepts and terms in 3D image based 3D treatment planning in cervix cancer brachytherapy with emphasis on MRI assessment of GTV and CTV. Radiother Oncol 2005; 74: 235-45.

39. Pötter R, Haie-Meder C, Van Limbergen E, Barillot I, De Brabandere M Dimopoulos J. et al. Recommendations from gynaecological (GYN) GECESTRO Working Group: (II): concepts and terms of 3D imaging, radiation physics, radiobiology, and 3D dose volume parameters. Radiother Oncol 2006; 78: 67-77.

40. Hellebust TP, Kirisits C, Berger D, Pérez-Calatayud J, De Brabandere M, De Leeuw AA, et al. Recommendations from Gynaecological (GYN) GEC-ESTRO Working Group: Considerations and pitfalls in comissioning and applicator reconstruction in 3D image-based treatment planning of cervix cancer brachytherapy. Radiother Oncol 2010; 96: 153-60.

41. Dimopoulos JC, Petrow P, Tanderup K, Petric P, Berger D, Kirisits C, et al Recommendations from Gynaecological (GYN) GEC-ESTRO Working Group (IV): Basic principles and parameters for MRI imaging within the frame of image based adaptive cervix cancer brachytherapy. Radiother Oncol 2012; 103: $113-22$.

42. Lindegaard JC, Madsen ML, Traberg A, Meisner B, Nielsen SK, Tanderup K, et al. Individualised 3D printed vaginal template for MRI guided brachytherapy in locally advanced cervical cancer. Radiother Oncol 2016; 118: 173-5.

43. Segedin B, Petric P. Uncertainties in target volume delineation in radiotherapy - are they relevant and what can we do about them? Radiol Oncol 2016; 50: 254-62.

44. Petrič P, Hudej R, Rogelj P, Blas M, Tanderup K, Fidarova E, et al. Uncertainties of target volume delineation in MRI guided adaptive brachytherapy of cervix cancer: A multi-institutional study. Radiother Oncol 2013; 107: 6-12.

45. Dimopoulos JC, De Vos V, Berger D, Petric P, Dumas I, Kirisits C, et al. Interobserver comparison of target delineation for MRI-assisted cervical cancer brachytherapy: application of the GYN GEC-ESTRO recommendations. Radiother Oncol 2009; 91: 166-72.

46. Petric P, Dimopoulos J, Kirisits C, Berger D, Hudej R, Pötter R. et al. Interand intraobserver variation in HR-CTV contouring: Intercomparison of transverse and paratransverse image orientation in 3D-MRI assisted cervix cancer brachytherapy. Radiother Oncol 2008; 89: 164-71.

47. Petric P, Hudej R, Rogelj P, Blas M, Segedin B, Logar HB, Dimopoulos JC. Comparison of 3D MRI with high sampling efficiency and 2D multiplanar MRI for contouring in cervix cancer brachytherapy. Radiol Oncol 2012; 46: 242-51.

48. Hellebust TP, Tanderup K, Lervåg C, Fidarova E, Berger D, Malinen E, et al. Dosimetric impact of interobserver variability in MRI-based delineation for cervical cancer brachytherapy. Radiother Oncol 2013; 107: 13-9. 
49. Petric P, Pötter R, Van Limbergen E, Haie-Meder C. Adaptive contouring of the target volume and organs at risk. In: Viswanathan AN, ed. Gynecologic Radiation Therapy: Novel Approaches to Image-Guidance and Management. Heidelberg: Springer, corp. 2011, p. 99-118.

50. Viswanathan AN, Erickson B, Gaffney DK, Beriwal S, Bhatia SK, Lee Burnett $O 3^{\text {rd }}$, et al. Comparison and consenus guidelines for delineation of clinical target volume for CT- and MR-based brachytherapy in locally advanced cervical cancer. Int J Radiat Oncol Biol Phys 2014; 90: 320-8.

51. Duane FK, Langan B, Gillham C, Walsh L, Rangaswamy G, Lyons C, et al. Impact of delineation uncertainties on dose to organs at risk in CT-guided intracavitary brachytherapy. Brachytherapy 2014; 13: 210-8.

52. Saarnak AE, Boersma M, Van Bunnigen BNFM, Wolterink R, Steggarda MJ. Inter-observer variation in delineation of bladder and rectum contours for brachytherapy of cervical cancer. Radiother Oncol 2000; 56: 37-42.

53. Petric P, Hellebust TP, Tanderup K, Poolakundan MR, Divakar SR, AlHammadi N, et al. Uncertainness of organs at risk delineation in MRI guided adaptive cervix cancer brachytherapy: A multi-institutional study. Eur J Cancer 2013; 49: S726.

54. Viswanathan AN, Dimopoulos JCA, Kirisits C, Berger D, Pötter R. CT versus MRI-based contouring in cervical cancer brachytherapy: results of a prospective trial and preliminary guidelines for standardized Contours. Int J Radiat Oncol Biol Phys 2007; 68: 491-8. 